\title{
Trends in the development of non-invasive technologies for assessing quality of seeds
}

\author{
Maksim Moskovskiy ${ }^{1}$, Aleksander Lavrov ${ }^{1 *}$, Anatoly Gulyaev ${ }^{1}$, and Maksim Sidorov ${ }^{2}$ \\ ${ }^{1}$ Federal Scientific Agro-Engineering Center VIM, 1 Institutsky proezd, 5, Moscow, 109428, Russia \\ ${ }^{2}$ Kaluga branch of the RSAU-Moscow Agricultural Academy named after A.K. Timiryazeva, \\ Vishnevskogo, 27, Kaluga, 248007, Russia
}

\begin{abstract}
The article presents studies on the use of non-invasive technologies to determine the quality of seeds of various crops. The authors analyzed patents on this topic. In particular, such laser and optical technologies as IR spectroscopy, hyperspectral spectroscopy, Raman spectroscopy, fluorescence, terahertz spectroscopy were investigated. The conducted patent research showed and confirmed the relevance of the work and the possibility of further implementation of the results into practice, mainly in the field of systems for laser-optical spectroscopy of seeds. Carrying out research on this topic will allow you to design and create new types of devices that will be in demand in many areas of modern agriculture.
\end{abstract}

\section{Introduction}

In ensuring the country's food security, the yield of agricultural crops plays an important role, which largely depends on the quality of the seed. In this regard, research is being actively carried out on the use of various physical methods for controlling the quality of seeds of agricultural crops.

The aim is to obtain information on the state of the art and statistically process selected titles of protection in the field of technologies and prototypes of laser-optical classifiers of seeds.

\section{Materials and methods}

Patent research covered the leading countries in the field of technologies and prototypes of laser-optical classifiers of seeds (Russia, USA, Japan, South Korea, China). Sources of information are publicly available [1-4]. The retrospective of the search in 20 years was determined by the terms of reference for an integral part Research Part on the topic «Development of environmentally friendly and energy efficient spectral and laser technologies to increase the productivity of agricultural plants and animals» according Agreement № 075-15-2020-774. The reliability of the results of patent research is ensured

\footnotetext{
*Corresponding author: vimlavrov@mail.ru
} 
in accordance with the list of indexes of classes of inventions to the IPC of the leading countries of the PCT (Patent Cooperation Treaty).

The content of the sections of the patent research report is determined in accordance with Russian standard GOST R 15.011-96 «System for the development and launch of products. Patent research. Content and procedure».

Determination of search countries, retrospective, sources of information, search indices, selection of information bases are made in accordance with the following recommendations [5].

Statistical processing of selected titles of protection in the field of technologies and prototypes of devices for assessing the quality of seeds based on spectral and laser technologies was carried out.

\section{Results and discussions}

It was analyzed titles of protection documents (patents) for the specified keywords and countries of patent holders in retrospect for the last 20 years.

The closest in nature are the following patents:

- Republic of Korea, Patent Invention, KR101424147B1, The system and the method for evaluating quality of seed;

- Republic of Korea, patent invention, KR1020030010025, Method for non-destructive analysis of one seed grain by near infrared ray absorption spectrum;

- USA, patent for invention, US2012092663A1, transmissionraman spectroscopy analysis of seed composition;

- Republic of Korea, patent for invention, KR20030019863A, Methodfordiscriminatingtheviabilityofseedsusingnearinfraredspectroscopy (Method for isolating seed viability by near infrared spectroscopy);

- USA, patent for invention, US2006160065A1, Method forclassifying plant embryos using Raman spectroscopy (Method for classifying plant embryos using Raman spectroscopy);

- USA, patent for invention, US2013229647A1, Method forclassifyingseeds, comprising the usage of infrared spectroscopy (Method for the classification of seeds, including the use of infrared spectroscopy);

- USA, patent for invention, US2009075325A1, Systems and methods for analyzing agricultural products;

- China, patent for invention, CN105224960A, Method for updating classification recognition model of corn seed hyperspectral image on the basis of clustering algorithm;

- Republic of Korea, patent for invention, KR101922447B1, Method for discriminating the cultivar of forage seeds using near-infrared spectroscopy;

- Russia, patent for invention, RU2016129306A, Method for the classification of seed.

The summarized results are presented in the graphs depicted in Figures 1-8. 


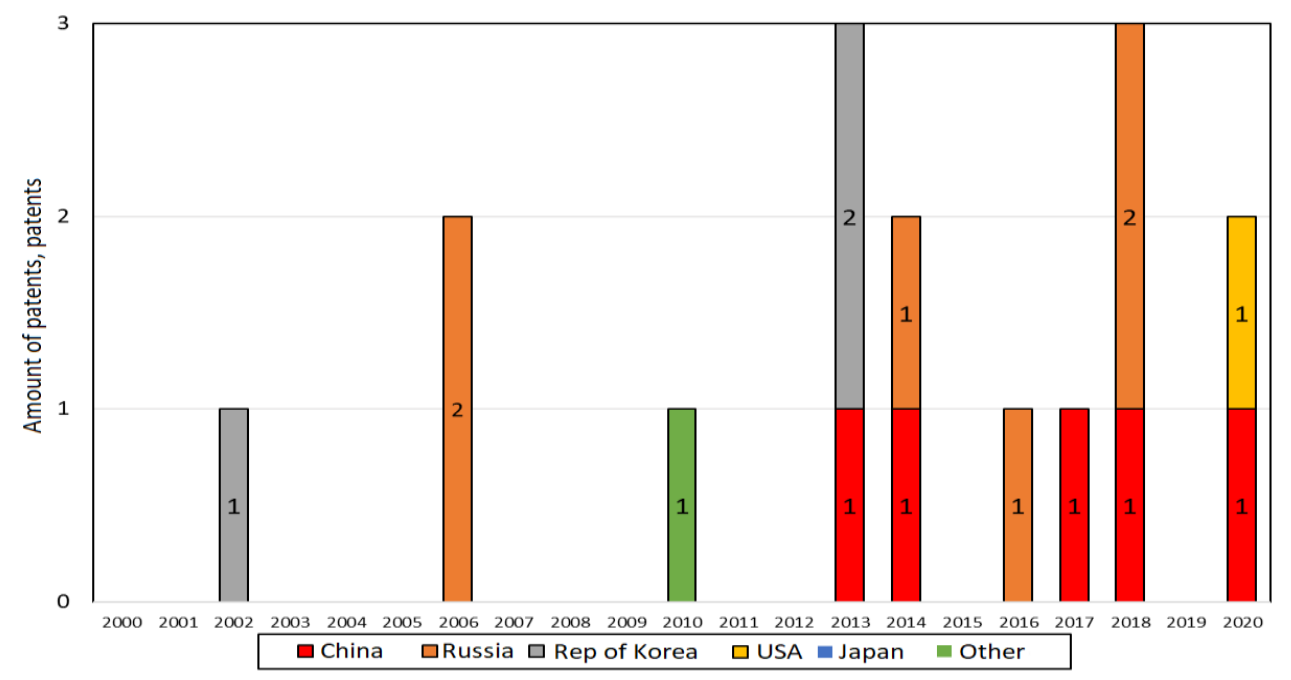

Fig. 1. Graph of the trend of patent activity of countries according to selected titles of protection: «Laser-optical classifiers»

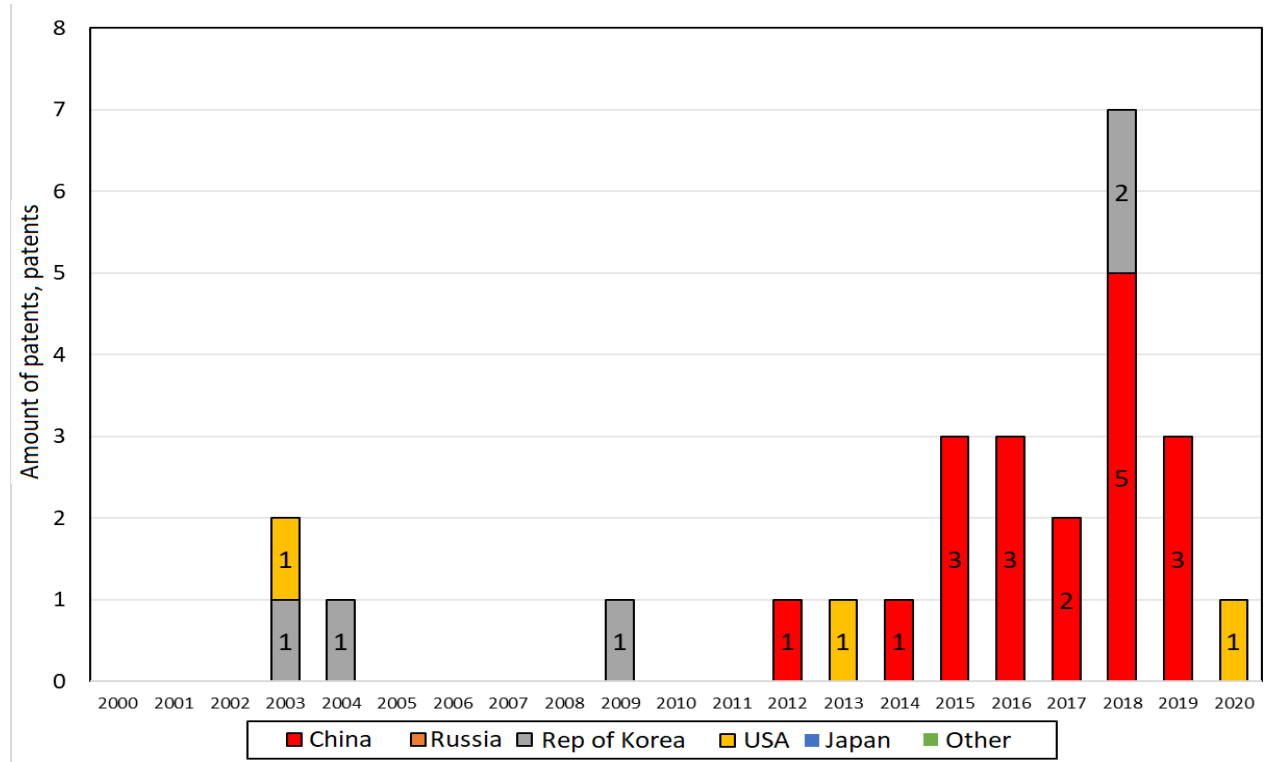

Fig. 2. Graph of the trend of patent activity of countries for selected titles of protection: «IR spectroscopy» 


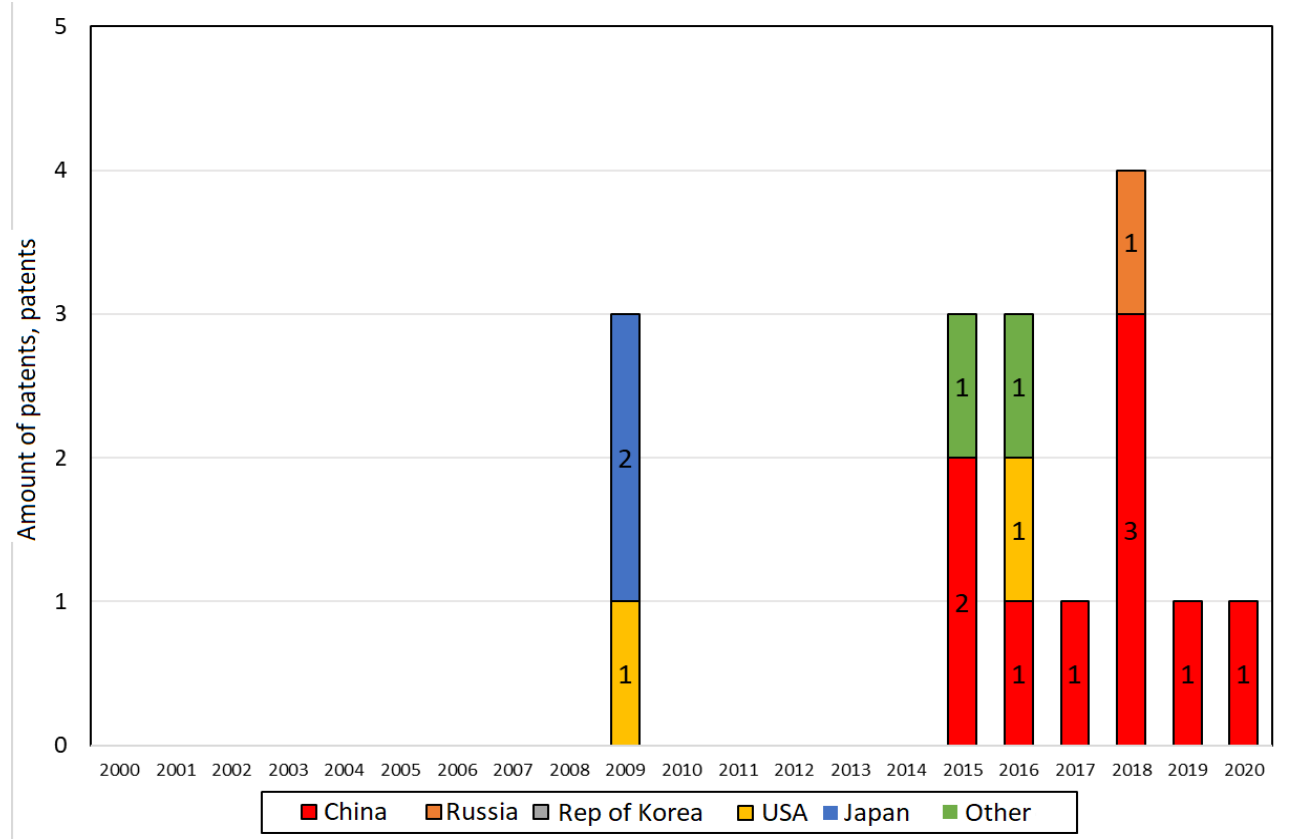

Fig. 3. Graph of the trend of patent activity of countries according to selected titles of protection: «Terahertz spectroscopy»

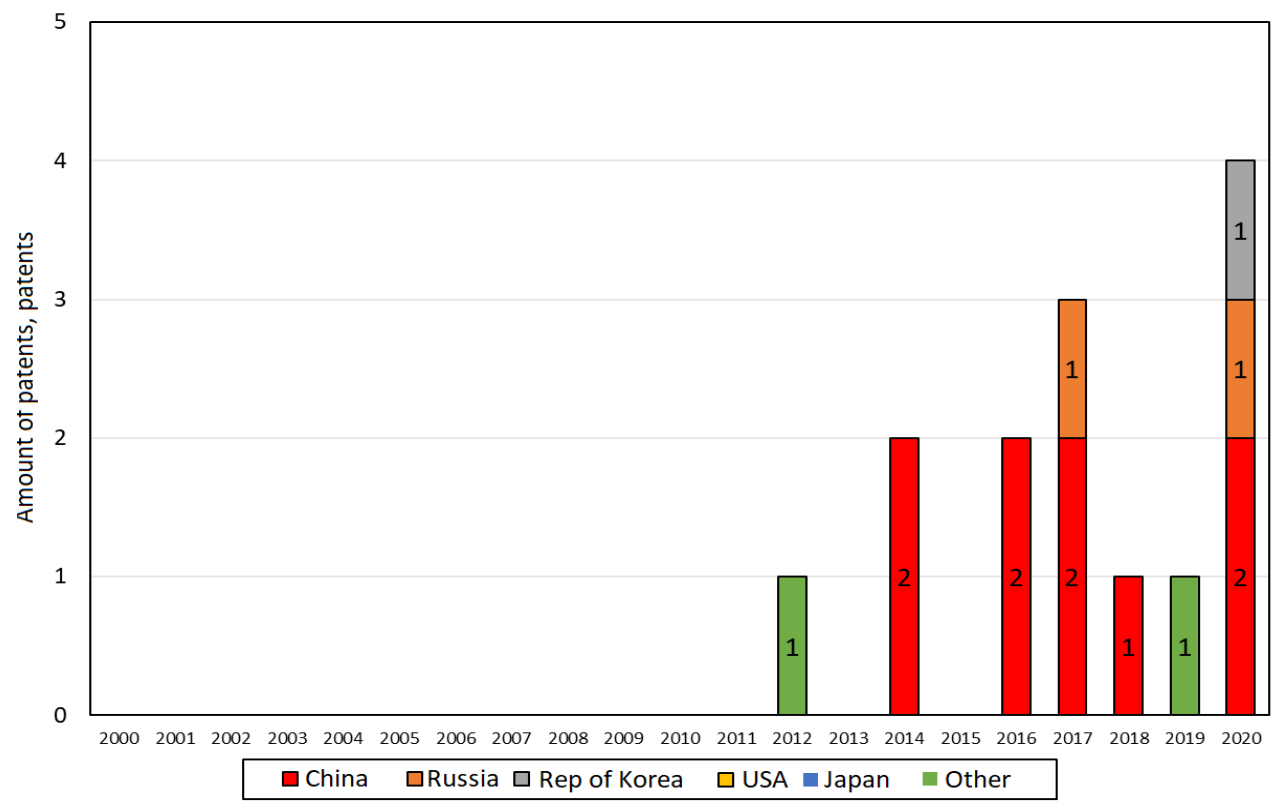

Fig. 4. Graph of the trend of patent activity of countries for selected titles of protection: «Hyperspectral image» 


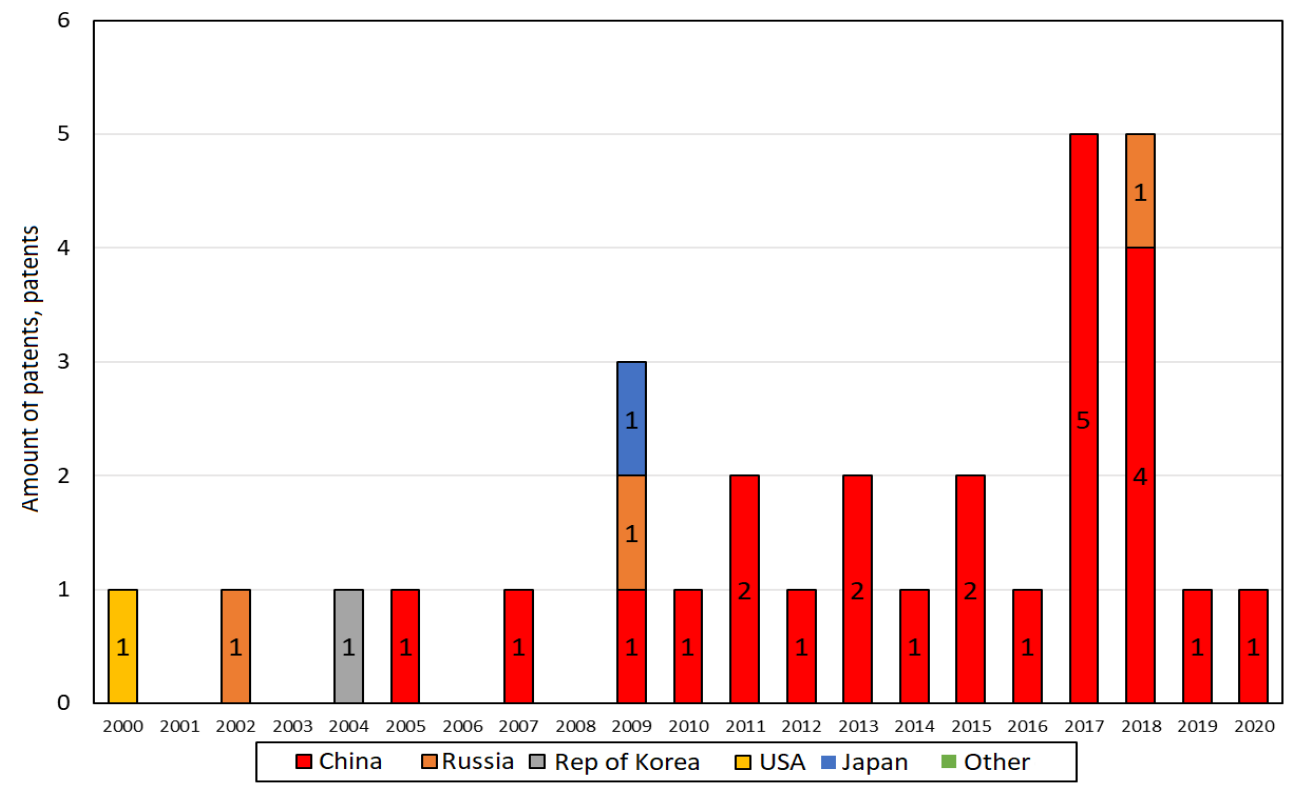

Fig. 5. Graph of the trend of patent activity of countries for selected titles of protection: «Fluorescence»

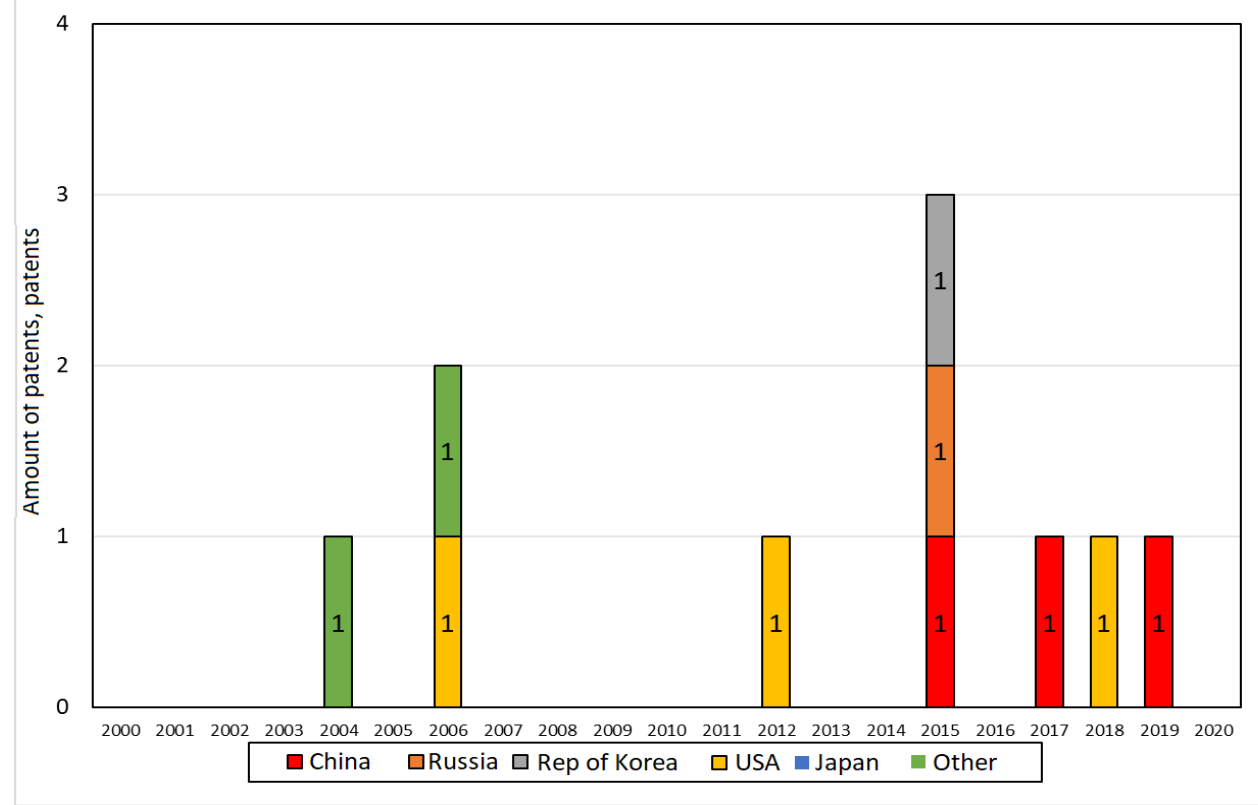

Fig. 6. Graph of the trend of patent activity of countries according to selected titles of protection: «Raman spectroscopy» 


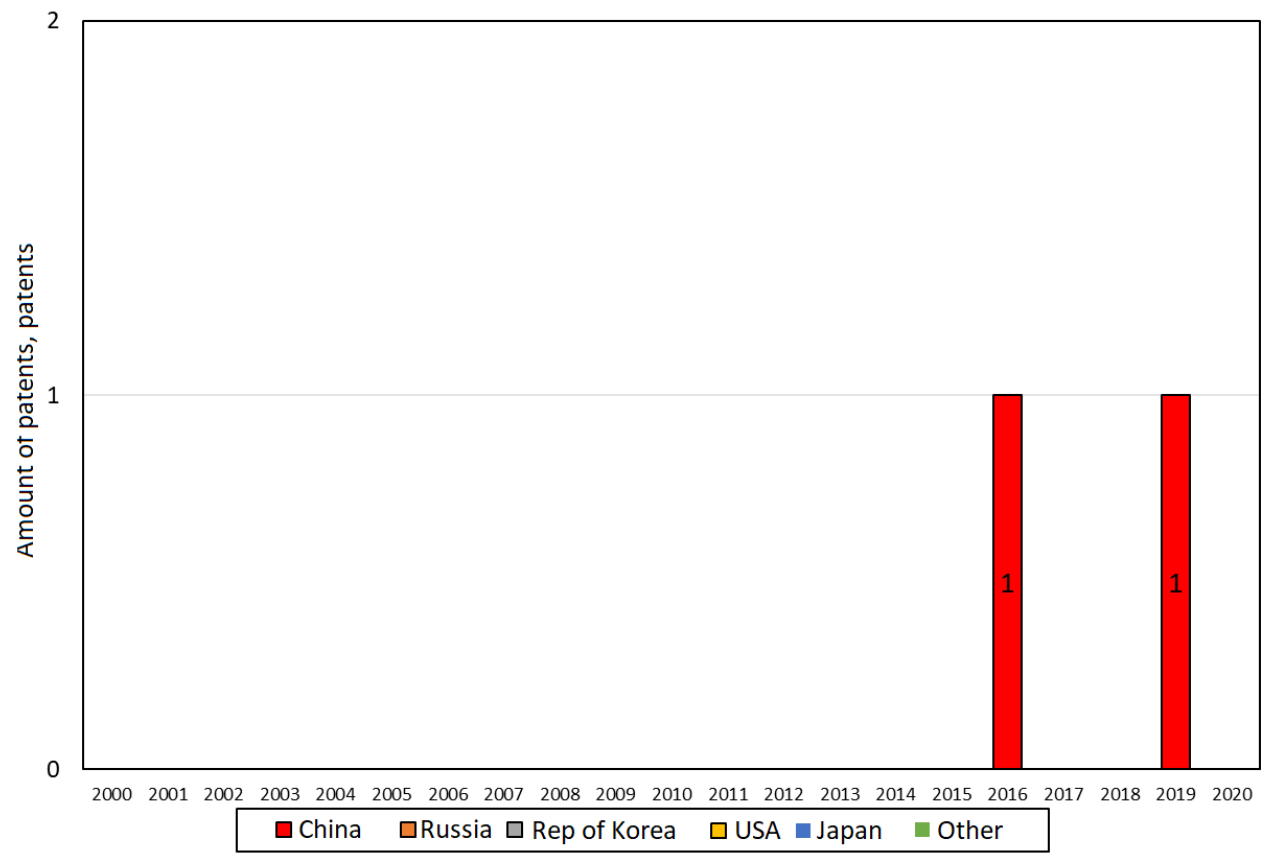

Fig. 7. Graph of the trend of patent activity of countries according to selected titles of protection: «Laser-induced breakdown spectroscopy»

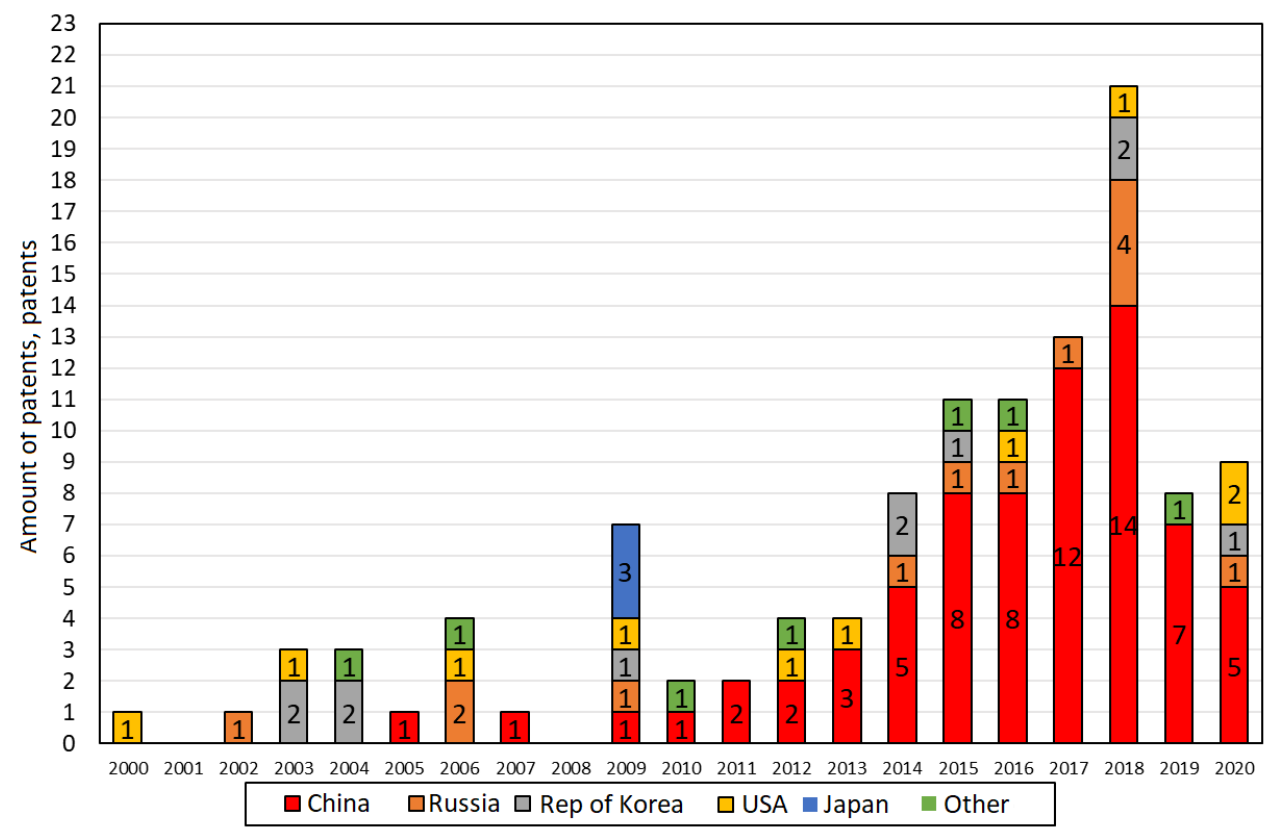

Fig. 8. Resulting graph of the trend of patent activity of countries for selected titles of protection: «Laser-optical methods of classification»

From the resulting graph (Figure 8), it can be seen that the number of patents related to optical spectroscopy methods is actively increasing, starting from 2009 to 2020, which indicates the probable development of technology in this direction. 
This is due to the fact that the inventions, which are based on the use of systems for laseroptical spectroscopy of seeds based on spectral and laser technologies, do not destroy the investigated object and allow research to be carried out quickly enough.

Figure 9 shows the distribution of patents by advanced country in the field of technologies and prototype devices for assessing the quality of seeds based on spectral and laser technologies.

\section{Amount of patents - 114}
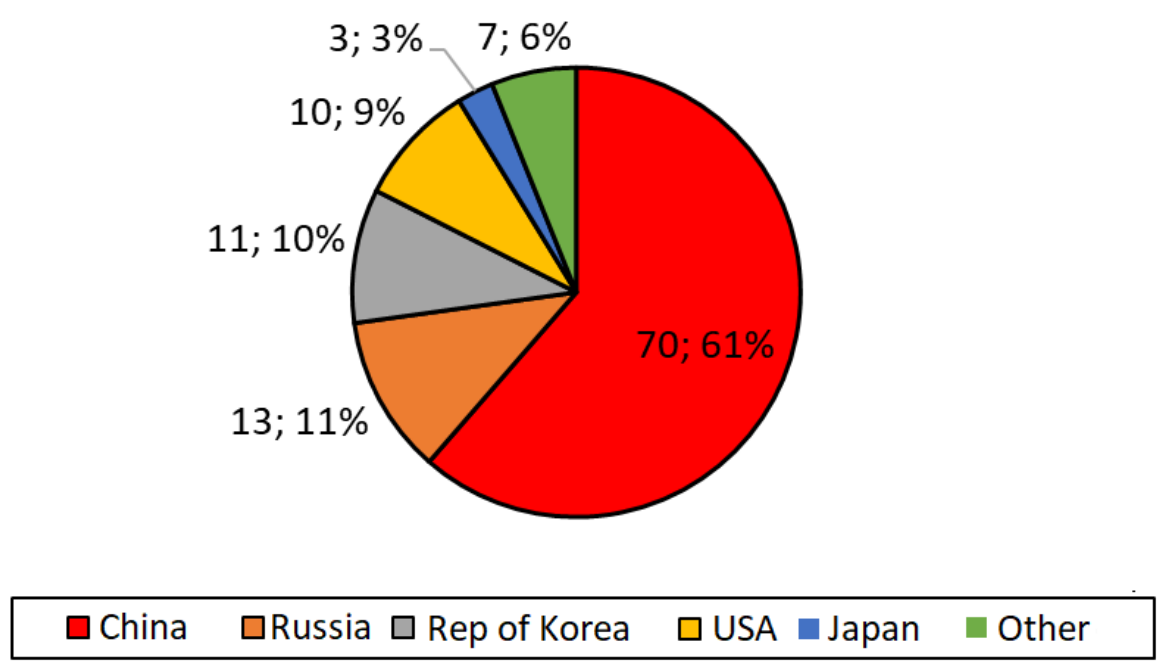

Fig. 9. Distribution of patents by country.

As can be seen in Figure 9, the main share of patents is registered in China - 61\%, in addition, the leading positions in the field of spectral and laser technologies are occupied by: Russia - $11 \%$, South Korea - 9\%, USA - $10 \%$.

\section{Concussion}

The conducted patent research showed and confirmed the relevance of the work and the possibility of further implementation of the results into practice, mainly in the field of systems for laser-optical spectroscopy of seeds. Carrying out research on this topic will allow you to design and create new types of devices that will be in demand in many areas of modern agriculture.

The research was carried out within the framework of agreement No. 075-15-2020-774.

\section{References}

1. https://new.fips.ru.

2. https://patents.google.com.

3. http://ep.espacenet.com.

4. www.orbit.com.

5. V.M. Medunetsky. Content and structure of patent research. - SPb: ITMO University, 2015 .-- 46 p. 